\title{
Secondary prevention of fractures after hip fracture: a qualitative study of effective service delivery
}

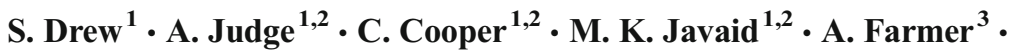 \\ R. Gooberman-Hill ${ }^{4}$
}

Received: 24 June 2015 / Accepted: 3 December 2015 / Published online: 12 January 2016

(C) The Author(s) 2016. This article is published with open access at Springerlink.com

\begin{abstract}
Summary There is variation in how services to prevent secondary fractures after hip fracture are delivered and no consensus on best models of care. This study identifies healthcare professionals' views on effective care for the prevention of these fractures. It is hoped this will provide information on how to develop services.

Introduction Hip fracture patients are at high risk of subsequent osteoporotic fractures. Whilst fracture prevention services are recommended, there is variation in delivery and no consensus on best models of care. This study aims to identify healthcare professionals' views on effective care for prevention of secondary fracture after hip fracture.

Methods Forty-three semi-structured interviews were undertaken with healthcare professionals involved in delivering fracture prevention across 11 hospitals in one English region. Interviews explored views on four components of care: (1) case finding, (2) osteoporosis assessment, (3) treatment initiation, and (4) monitoring and coordination. Interviews were
\end{abstract}

S. Drew

sarah.drew@ndorms.ox.ac.uk

1 Oxford NIHR Musculoskeletal Biomedical Research Unit, Nuffield Department of Orthopaedics, Rheumatology and Musculoskeletal Sciences, University of Oxford, Windmill Road, Headington, Oxford OX3 7LD, UK

2 MRC Lifecourse Epidemiology Unit, University of Southampton, Southampton General Hospital, Southampton SO16 6YD, UK

3 Nuffield Department of Primary Care Health Sciences, Radcliffe Observatory Quarter, Oxford OX2 6GG, UK

4 School of Clinical Sciences, University of Bristol, Learning and Research Building, Level 1, Southmead Hospital, Bristol BS10 5NB, UK audio-recorded, transcribed, anonymised and coded using NVivo software.

Results Case finding: a number of approaches were discussed. Multiple methods ensured there was a 'backstop' if patients were overlooked. Osteoporosis assessment: there was no consensus on who should conduct this. The location of the dual energy X-ray absorptiometry (DXA) scanner influenced the likelihood of patients receiving a scan. Treatment initiation: it was felt this was best done in inpatients rather request initiation in the post-discharge/outpatients period. Monitoring (adherence): adherence was a major concern, and participants felt more monitoring could be conducted by secondary care. Coordination of care: participants advocated using dedicated coordinators and formal and informal methods of communication. A gap between primary and secondary care was identified and strategies suggested for addressing this.

Conclusions A number of ways of organising effective fracture prevention services after hip fracture were identified. It is hoped that this will help professionals identify gaps in care and provide information on how to develop services.

Keywords Epidemiology $\cdot$ Fracture $\cdot$ Fragility $\cdot$ Hip · Osteoporosis $\cdot$ Qualitative

\section{Introduction}

About 87,000 hip fractures occur annually in the UK and are estimated to cost around $£ 2.3$ billion annually including health and social care [1]. These fractures usually occur in individuals with underlying osteoporosis [2,3], and these are at high risk of subsequent fractures [4] and premature death [5].

Bone protection therapies reduce the risk of subsequent fractures [1, 6-8], and guidance has been published on how best to organise services to prevent further fractures $[1,8-10]$. 
According to the International Osteoporosis Foundation, services should be structured around four aspects of care: case finding; osteoporosis assessment, including a dual energy Xray absorptiometry (DXA) scan to measure bone density if needed; treatment initiation with bone protection therapies for those with osteoporosis; and falls risk assessment and systems to monitor adherence to therapies [11].

To coordinate these aspects of care, services with named clinicians responsible for coordinating the multi-disciplinary teams, known as coordinator-based systems, are recommended [12]. However, there is no consensus on the best model of care, and there is significant variation in the processes used by each hospital to undertake the four main components of a fracture prevention service and the types of coordinatorbased models in place [13].

This article identifies the elements of care of hip fracture patients that health professionals think are most effective in preventing secondary fractures after hip fracture. This includes the processes for undertaking the four main components of a fracture prevention service and coordination of care. It is hoped that this study will help inform clinicians and service managers about which fracture prevention services to develop in the future.

\section{Methods}

Qualitative methods were chosen as the most appropriate means to elicit the views and experiences of professionals working in fracture prevention services [14]. To collect information from busy professionals, to structure data collection around existing knowledge and to expedite analysis, the study design comprised one-to-one interviews [15] focusing on main components of fracture prevention and ways of coordinating care [11]. In the analysis described here, we use a thematic approach [16]. The findings here provide information about the delivery of fracture prevention services in practice, complementing our theoretically driven abductive analysis exploring the implementation of fracture prevention services presented elsewhere [17]. Elements of study design and conduct are described in turn.

\section{Sample}

Professionals involved in providing services to prevent secondary fractures after hip fracture in the 11 hospitals that receive acutely hip fractured patients in one region in England were approached to take part in this qualitative interview study. Potential participants were identified by a clinical lead champion in osteoporosis and an operational service manager in trauma who were both working within the Region. Potential participants were then purposively sampled to ensure that participants were drawn evenly from the 11 acute trusts and included a range of professionals, who were orthogeriatricians, fracture prevention nurses, trauma nurses, hospital practitioners in osteoporosis, surgeons and service managers. In three waves of recruitment, we approached potential participants by email, followed up by an additional email 2 weeks later and a subsequent telephone call. During the process of recruitment, we also used snowball sampling [18] and approached other professionals who were suggested by participants. In total, 82 professionals were contacted to take part in the study and 43 agreed to do so. The remaining 39 were either unavailable or did not wish to take part.

\section{Ethics}

Ethical approval was provided by the Central University Research Ethics Committee (CUREC) in 2012, reference number MSD-IDREC-C1-2012-147. Each involved NHS trust provided R\&D approval.

\section{Interview procedure}

Participants provided their written, informed consent before interview. Consent included their agreement to audiorecording of interviews and publication of anonymous quotations. Interviews took place in 2013 at a time and location convenient to the participant and lasted 30-50 min each. Most interviews were conducted face-to-face, and one interview took place by telephone. Using a topic guide to ensure that she covered key topics with all participants, the researcher asked open-ended questions about participants' views and experience of best models of care for the prevention of secondary fractures after hip fracture. Interviews included focus on the four main components of a fracture prevention service and the best ways of coordinating care [11].

\section{Data analysis}

Interviews were audio-recorded, transcribed, anonymised and imported into the qualitative data analysis software NVivo. A thematic analysis was conducted by the project researcher, which involved coding the interviews to identify themes and subthemes in the responses [16]. Twenty percent of the interviews were independently coded by another member of the team (RG-H). The coding frame was then discussed and refined by the two researchers. The themes and subthemes which were identified were then transferred onto charts using a framework approach to data organisation [19]. This allowed the project researcher to compare and contrast the responses of different participants. Descriptive accounts were then generated by exploring the themes and subthemes that had emerged. 


\section{Results}

\section{Characteristics of participants}

The 43 participants comprised eight fracture prevention nurses, four orthogeriatricians, four geriatricians, two general practitioner (GP) osteoporosis specialists, five consultant trauma orthopaedic surgeons, eight rheumatology consultants, two orthopaedic nurses, one trauma matron, one matron for the hip fracture unit, one falls coordinator, one falls nurse, one bone densitometry specialist and five service managers. Between three and seven participants were drawn from each of the 11 hospitals. Years of experience in their current role ranged between 8 months and 32 years and time spent working at the hospital between 1 and 27 years. We found that the views of different groups of healthcare professionals did not differ to any great degree, and as a result, we do not distinguish between them in our findings.

The experiences and views of healthcare professionals and service managers on the most effective ways of preventing secondary fractures after hip fracture are summarised below. This includes the processes for undertaking the four main components of a fracture prevention service [11] and coordination of care (Fig. 1).

\section{Case finding (Box 1)}

Participants were asked to discuss their experiences of identifying hip fracture patients at risk of secondary fractures: 'case finding'. Participants felt that such patients were relatively easy to identify since they were invariably admitted and remained in hospital for a period of time and therefore presented a 'captive audience'. This was made easier when patients were located on one ward such as dedicated hip fracture or trauma units as 'outliers' could be missed.

Participants felt it was important to have a named clinician responsible for case finding rather than trauma surgeons and to establish a systematic process of case finding with them. The relative advantages and disadvantages of different methods were discussed. Computerised databases provided clinicians with information about patients which helped them to undertake osteoporosis assessments. However, 'human error' meant that data was maybe inputted incorrectly or patients missed which limited its utility.

Attendance of fracture prevention coordinators or other healthcare professionals at daily pre-operative trauma meetings was seen as effective at identifying patients at risk of further fractures. This was despite awareness that such attendance involved listening to extraneous information and relying on clinicians including discussion of all relevant patients. Perioperative or post-operative ward rounds were successfully used to identify patients although participants were concerned


Fig. 1 Summary of healthcare professionals' and service managers experiences and views on the most effective ways of preventing secondary fractures after hip fracture is identified in this study 
that patients seen perioperatively could be missed if they were particularly ill.

Relying solely on trauma meetings or ward rounds was problematic when staff were absent as it meant that there was no one to identify cases. To mitigate this, participants emphasised the importance of back-up such as computerised databases to identify patients retrospectively. Multiple methods were also used to provide a 'backstop' if patients were overlooked, 'someone who picks up, sweeps up behind'.

\section{Osteoporosis assessment (Box 1)}

There was consensus that osteoporosis assessments were best done in an inpatient setting where possible to enable clinicians to initiate bone protection therapies more quickly. Participants believed that there was a risk of 'losing' patients after discharge as at that time, they may not receive appointment letters, may be too frail or forget to attend appointments.

Participants discussed the advantages and disadvantages of the assessment being carried out by orthogeriatricians and fracture prevention nurses. Orthogeriatricians were thought to have the skillset to assess more complex patients. However, some thought that this was an expensive model of care and a large workload for clinicians who were also responsible for carrying out falls assessments and optimising patients for theatre. Participants said patients saw fracture prevention nurses as approachable and thought they had more time to spend with patients than other professionals. These were thought to mean that patients would provide better information to fracture prevention nurses. This view was shared by participants from all professional groups, and two fracture prevention nurses felt that it could be useful for them to undertake a nurse prescribing course. Other participants had found a nurse-led model with medical back-up from orthogeriatricians or metabolic bone physicians was effective. Rheumatologists were also seen to have a role in providing more specialized advice when required on treatments such as in the first few months when denosumab was available, atypical osteoporosis and less common secondary causes of osteoporosis.

Participants discussed the advantages and disadvantages of using a pro forma when assessing patients. In straightforward cases, they felt it enabled them to ensure that the assessment was performed thoroughly and consistently. Others thought it meant patient-specific factors could be overlooked and that questions should be tailored to individual patients.

There was no consensus on the best time to conduct assessment. Two participants felt that $48 \mathrm{~h}$ post operatively was long enough to give patients time to recover from immediate effects of the operation, and others felt that 4 or 5 days post operatively was more appropriate. Most advocated flexibility depending on the needs of individual patients. However, the timing of the assessment was often constrained by the capacity of the service. To assess cognitively impaired patients, participants obtained assessment information from close relatives or initiated treatment without a full assessment.

\section{DXA scanning}

The majority of participants were of the opinion that services should adhere to NICE guidelines by providing DXA scans to those aged under 75 and treating those aged $\geq 75$ without the absolute need for DXA [20]. Participants' reasons for this included the high levels of osteoporosis in those aged $\geq 75$ and finite resources in the NHS. However, participants were also flexible in this, for instance when patients $\geq 75$ needed to be presented with 'proof' before they would agree to start taking treatments. One interviewee was concerned that the NICE guideline meant some patients who would benefit from a DXA scan were being overlooked.

The location of the DXA scan, whether in an inpatient, outpatient or community hospital, was seen to impact on whether patients received it. The majority of participants felt that it was most appropriate to administer it in an outpatient setting. Nevertheless, one participant favoured an inpatient setting since it meant he could ensure patients received a scan and enabled him to initiate treatment more quickly. However, he acknowledged that scan provision was dependent on whether patients were well enough.

Conducting a scan in the post-discharge outpatient setting gave patients time to recover from their operation. But this idea was tempered by concern about failure of patients to attend appointments. This was seen as being particularly problematic when patients lived a long way from the scanner. Strategies for mitigating this were ensuring that the scanner was accessible by having good public transport links and providing transport to those who were not able to reach the hospital. To encourage patients to attend appointments, one participant thought it useful to emphasise the importance of a scan to patients whilst they were still receiving inpatient care. Another participant initiated treatment on the same day as administering the scan so that patients only had to attend one appointment and sent reminders prior to appointments.

The process of referral to scans was seen as important. Whilst some orthogeriatricians and fracture prevention nurses described how they were able to refer patients directly, others had to request an appointment via primary care. Interviewees felt the latter approach was problematic as it meant patients had to wait longer, delayed the start of treatment and made it more likely that patients would 'get lost' in the system. Methods of referral were constrained by contracting arrangements with primary care which some found frustrating. 
A number of participants also highlighted the lack of capacity to refer patients to be DXA scanned and wanted more frequent access or additional scanners in place.

Box 1 Case finding and osteoporosis assessment

\section{Case finding}

It depends which doctors have been on the night before as to how much has been put onto [the computer system]... some of it turns out to be rubbish [Participant ID: 010]

\section{Osteoporosis assessment}

I'd love to see a nurse specialist in there because I think some of those older people are far less intimidated... to see a fracture nurse in that setting actually exploring what those answers mean, getting the detail would be brilliant. [Participant ID: 016]

You know breaking your hip is huge, there's lots of psychological things... Being flexible about [the timing] is probably a bit more patient-centred. [Participant ID: 029]

\section{DXA scanning}

It really depends how able they are to get on and off the bed and stick their legs in the air and that sort of thing. [Participant ID: 008]

I think having it done as an Outpatient is more appropriate because most of them are generally quite sick or unwell or they're still in pain so it would be difficult to get them to the DEXA. [Participant ID: 019]

Our biggest barrier is obviously the fact that we have to drag our patients from [another town] down to [the city]. [Participant ID: 004]

We should just be able to refer directly... It just adds in an additional communication where we know there's problems, where referral forms get missed, go missing, patients fail to attend their appointment and no one follows that up. [Participant ID: 030]

The powers that be... don't like us DEXA-ing people internally, because they like the money flow, so they prefer them to go out and then come back. [Participant ID: 009]

\section{Treatment initiation (Box 2)}

Aged 75 and over

Participants were asked to discuss their experiences of initiating a range of bone protection therapies including bisphosphonates, raloxifene, strontium ranelate, denosumab and teriparatide $[8,20,21]$. They felt that patients who were $\geq 75$ and who did not need a DXA scan should have their treatment initiated in an inpatient setting where possible. This meant they could begin treatments more quickly and enabled clinicians to assess whether they could tolerate it. However, some clinicians were concerned that patients were sometimes too 'shocked' post operatively to understand how and why they were taking the therapies which could impact on adherence. They therefore stressed the need to follow this information up post-discharge. Others were worried that treatments were being initiated before clinicians could get a 'feel' for their prognosis.

Some participants were worried that patients were not being prescribed treatments whilst they were inpatients. One clinician had found it useful to put 'checks' in place so that their colleagues were able to spot if they had not been prescribed. Furthermore, treatments were not always included on discharge summaries. To mitigate this, participants thought it could be useful to build automated reminders into electronic patient records or use more senior staff to write summaries. There was also a concern that discharge summaries were not always read by GPs. To address this, one participant had replaced formal letters with a shorter, more concise report.

\section{Aged under 75}

For those aged under 75 who received a DXA scan or who needed treatments that could not be initiated immediately [20], therapies were either initiated in primary care or in an outpatient setting. However, there was a concern that this was not being done consistently in primary care and that GPs lacked 'alertness' about the importance of the therapies.

To help GPs make appropriate treatment decisions, healthcare professionals in secondary care often sent treatment recommendations and DXA reports. Improvements in written communication were suggested to encourage GPs to read them. One participant thought it would be useful to issue pro formas to GPs to help them make decisions. In one service, healthcare professionals had issued written guidelines but had struggled to ensure they were accessible enough for GPs to use them regularly.

\section{Monitoring (Box 2)}

Of the areas explored, participants were most concerned about the low levels of adherence to bone protection therapies and felt that monitoring had the potential to improve adherence to oral therapies. Since bisphosphonates are generally the first line of treatment for osteoporosis in the UK [8], a number of participants thought that zoledronic acid should be made more widely available. They felt this could help improve adherence since it is given at a clinic rather than at home and given once a year rather than being taken regularly. However, they also described how the provision of this therapy was often constrained by local guidelines.

\section{By primary care}

Patients prescribed oral bisphosphonates were generally monitored in primary care. However, there was a worry that this management was often 'sub-optimal' and some participants were concerned that GPs did not always have enough knowledge to monitor patients effectively.

Participants thought that the introduction of the Quality Outcomes Framework in primary care [22] was of limited use since it did not provide a large enough financial incentive 
to primary care to deliver effective services. To address these problems, participants thought it would be useful to introduce osteoporosis champions and fracture prevention nurses into primary care to provide GPs with support and guidance in setting up and delivering services. They also thought 'open communication' was important and used letters, emails and phone calls to communicate with GPs. However, one participant felt that these were only utilised by those who were interested in the disease.

\section{By secondary care}

On account of these problems, participants thought that more monitoring could be conducted by secondary care. However, there was no consensus on how this could be systematically achieved. Participants discussed the relative advantages and disadvantages of using questionnaires, telephone calls and outpatient's appointments to perform monitoring.

Questionnaires were used as a 'prompt' to call patients or GPs if a problem was identified and were not time consuming to administer. However, participants felt relevant information may be missed out and had experienced a 'poor' return rate. Contacting patients by phone allowed participants to ask 'prompting questions' to obtain information. Because of this, participants felt it was best done by someone with clinical knowledge. However, there was no capacity for this type of followup by some services. Others wanted to follow up patients in outpatient clinics as it was 'easier to assess patients face-to-face'. However, most felt it was unnecessary and lacked the resources to do so.

Some participants had found it difficult to contact nursing homes as staff were busy and therefore difficult to get hold of. In these cases, they thought it might be easier to use questionnaires that they could complete in their own time. Another undertook a 'ward round' so he could obtain information from relatives or members of staff.

Participants felt the purpose of monitoring was to reiterate the importance of adherence, ensure that this was being done properly and to clarify instructions if necessary, and identify any problems such as swallow issues that meant other therapies such as zoledronic acid were more appropriate.

Since there was limited capacity for follow-up in many of the services, the majority of participants prioritised follow-up between 6 and 12 weeks as it enabled them to 'catch' those who were not adhering and 'give them a second go'. It was felt this was a suitable length of time to revisit the information provided on discharge before it had been forgotten. Some participants also suggested targeting individual patients. One participant felt it was important to be flexible with this depending on the needs of individuals.
Box 2 Treatment initiation and monitoring (to enhance adherence)

\section{Treatment initiation}

Aged 75 and over

I don't think there's an option for it to be initiated in Primary Care for us at the moment because even when we send people home on it the GPs don't always continue it [Participant ID: 017]

It's not missed because if I missed it the nurses would pick up... the Fracture Liaison Nurses would pick it up or the Pharmacist would, you know there are various checks that they wouldn't go out without their bone protection. [Participant ID: 005]

GPs get probably 400 or 500 letters a day, do they read everything? Hopefully they do. [Participant ID: 035]

Aged under 75

[We] don't know how many have treatment initiated [by GPs] [Participant ID: 017]

It's almost like [GPs] need a package of almost instructions with a tick box... so that yeah it's straight forward for them. [Participant ID: 037]

I think that there are sort of conflicts in having guidelines that are actually useful in making an individual treatment decision whilst being sufficiently simplistic enough for someone to be able to commit to memory and remember. [Participant ID: 024]

GPs are fantastic, but how can they be experts and know everything... And that's why I think we have a duty to them and to our patients to inform appropriately. [Participant ID: 042]

\section{Monitoring}

We know that compliance is the issue, and we know that less than $50 \%$ are taking them at the year mark. [Participant ID: 010]

\section{By primary care}

This has to be owned by Primary Care, I mean this is a long term condition, osteoporosis and fracture prevention, and GPs are responsible for managing long term conditions [Participant ID: 026]

I think in the hospital we have to remember that these are the GP's patients not our patients... we have to put responsibility onto the GP and onto the patients to make sure that they do take their tablets. It's a bit buck passing really but that's how it needs to be [Participant ID: 002]

Well in theory the GPs should be monitoring these patients... But it doesn't happen. It might happen on the odd GP, but that isn't happening [Participant ID: 011].

The GPs that liaise with you are probably the GPs that you're not worried about because they are trying to improve their knowledge; it's the ones that you never hear about that never call you that think they can manage this condition that they can't [Participant ID: 020]

\section{By secondary care}

The patients didn't realise [taking the medication] was that important, I don't think. Whereas if they're getting a phone call at three months to check they're going to think, "Well it must be important because they're phoning me to see if I'm taking the tablet. Oh I'd better carry on taking it. [Participant ID: 010]

It was a waste for them coming in, ten minute appointment, and it was a waste of a clinic slot that could be done for someone that is struggling or not responding or needing treatment. [Participant ID: 042]

Usually [undertaking a ward round in a nursing home] is simpler than the patient coming here and if the patients come here they usually come with an escort who doesn't have additional information, whereas if we go there the patient has the nurses that have been looking after the patient and we get a lot more information from them that way. [Participant ID: 038] 
I would like far more time to be able to follow these patients up more thoroughly [Participant ID: 002]

I just don't have the resource to [follow-up] [Participant ID: 023]

Six months down the line, the horse has already bolted and they won't remember what you said beforehand, so I think three months is at least the initial thing [Participant ID: 024]

[6 - 12 weeks is] a nice length of time to revisit all the information you gave them at the diagnosis, and make sure that they're understanding everything, and that you can go through the lifestyle again... I think any sooner would be too quick, and maybe a bit longer is too long. [Participant ID: 042]

So [monitoring] doesn't become so unwieldy... identify your patients that you really need to follow up and identify the patients that you may [Participant ID: 028]

It's more that you need flexibility within the service to be able to account for the differences in individuals. [Participant ID: 024]

\section{Coordination of care (Box 3)}

Participants discussed their experiences of coordinating the multi-disciplinary teams involved in fracture prevention.

\section{Coordinator-based models of care}

All interviewees advocated using a dedicated coordinator to coordinate the multi-disciplinary teams, whether this be a fracture prevention nurse or consultant-led service. Participants felt it was useful to have a coordinator that 'know[s] who's who and who to contact' and who could provide a link to other wards if it was necessary. They also felt coordinators provided 'continuity of care' for patients which helped them to build more trusting relationships. Some thought it was best to have a number of clinicians responsible for this so that if a colleague was absent, there was someone who could fulfil this role.

\section{Additional strategies for coordinating care}

Other strategies for coordinating care were discussed. Participants felt that multi-disciplinary paperwork such as protocols and pro formas were useful as they meant colleagues had access to all the information that could help them make a treatment decision and that tests were less likely to be duplicated. Some thought that electronic patient records were the most efficient way of transferring this information. Joint ward rounds were also used and ensured that no information was forgotten when it was transferred to colleagues. Regular multidisciplinary meetings helped to ensure that the team communicated regularly and enabled colleagues to develop a 'mutual professional respect'. Informal communication was also seen to be important.

\section{Coordination of care between primary and secondary care}

Participants felt that there was a lack of communication and cooperation between primary and secondary care. Training sessions had been used to try and address this. However, although some had found them useful, others felt that 'you only get the ones that are interested' and had cancelled sessions due to a lack of interest. Other participants thought it might be useful to develop local guidelines with GPs and contact them for feedback on their services.

Box 3 Coordination of care

Are they having that continuity of care, are they having a regular orthogeriatric input? No, and I think that's something that we can improve upon [Participant ID: 006]

\section{Coordinator-based models of care}

It needs a strong lead, it needs a me or equivalent of me really... In terms of the Orthopaedic Team, you know you're working with Orthopaedic Nurses so it's a different culture set... So you need to sort of pull in the ethos [Participant ID: 026]

There is a communication pathway which we never had before [Participant ID: 008]

You often establish relationships and people can become more trusting and they can tell you if they're actually taking their medications or not [Participant ID: 022]

Well the only problem we have is the service is so good when she's here; it's a problem when she's not. [Participant ID: 001]

\section{Additional strategies for coordinating care}

It's much easier to communicate with people that are on the end of the phone and because we have meetings every month [Participant ID: 009]

It's just about encouraging people to know that they all have a role, we all have a responsibility to deliver the quality care and all of us are important in making that so. You know each of you can't do it without the other and it's actually about ownership and responsibility [Participant ID: 026]

\section{Coordination of care between primary and secondary care}

There seem to be very - two separate camps: there's what actually happens in trauma and then there's what happens in primary care, and the communication is difficult. [Participant ID: 009]

I don't think it's very good at all... its very much them and us isn't it situation. [Participant ID: 002]

It's about making sure GPs are happy with that and they are happy because they've been involved in the decision making. [Participant ID: 002]

I mean it is a big hole really, we ought to find out what they think of the discharge summaries and things... I suppose I need to go back to the GPs and say "well what do you think of this service now? [Participant ID: 004]

\section{Discussion}

This study supports existing guidance and research on the best models of care for the prevention of secondary fractures. This includes the value of using a dedicated clinician to perform 
case finding [1], provision of DXA scans to those aged under 75 years and initiating treatment for those aged $\geq 75$ without a DXA [20]. Using fracture prevention nurses as fracture prevention coordinators with medical back-up, which was advocated by a number of participants, is also the model proposed by the Department of Health [23]. Concern about the low levels of adherence to bone protection therapies reflects findings from previous studies [24]. Although no studies have examined adherence to zoledronic acid compared to oral therapies, the high levels of adherence to zoledronic acid has been demonstrated [25] along with patient preference for this therapy [26]. More recent data has confirmed high rates of adherence to denosumab [27]. The use of monitoring to improve adherence is also well established [28]. To coordinate the multi-disciplinary teams involved in fracture prevention, there is also an international consensus about the need for coordinator-based models of care [11, 12, 29-31]. This study expands on this by exploring participants' views on the best ways of enacting these guidelines and the challenges they encountered in doing so. Surprisingly, there was no consensus on the best time to conduct assessment. One of the biggest issues identified was the failure of primary and secondary care to communicate effectively, especially with regard to treatment initiation in primary care. Issuing guidance on these would provide clarity and help clinicians to address these challenges. Participants also challenged guidelines which suggest GPs are best placed to monitor adherence to treatments [1] and thought more could be done by secondary care. Since effective fracture prevention is a global concern [11], it is possible that these findings may be usefully applied to other care settings. It is worth noting that although we recognise the nature of the biological assessments being carried out prior to treatment initiation is crucial, we felt this subject was best suited to clinical studies.

A qualitative study design using interviews enabled us to identify and explore the views and experiences of professionals working in the area of fracture prevention. Employing open-ended questions but focusing on known areas for fracture prevention was designed to enable professionals to speak candidly about their experiences. The sample comprised 43 participants, who were purposively sampled to ensure that all professional groups were represented. The study did not seek to achieve saturation as in some qualitative research but sought to achieve an appropriate sample providing adequate representation of involved professionals [32]. Unless appropriate, we do not distinguish between the views of different groups of professionals working within the field as we found that there was little difference in their perspectives except that clinically trained staff were able to reflect on clinical practice, whereas it was not the place of managers to do so. The study only took place in one region, and although it included all NHS trusts in that region, further work may be needed to explore fracture prevention services in other areas.
Furthermore, it is possible that participants' experiences of working with primary care may not reflect the perceptions of GPs, which could be the subject for a further study.

The study has successfully explored the best models of care for the prevention of fractures after hip fracture. Identifying these will help inform clinicians and service managers about how to develop fracture prevention services in the future. Further work could explore the delivery of fracture prevention services in primary care. This would offer a 'system-wide' perspective which would over arch the division between primary and secondary care. Further research could also explore the experiences of patients with hip fracture and their significant others of accessing these services to add a 'patient-centred' context to the organisation of services. In addition, whilst the study focused on fracture prevention rather than falls prevention services, we acknowledge that these are interrelated [1] and further work could be conducted on this area. There is also a clear need to understand the most effective ways of monitoring patients to improve adherence to ensure that they experience the full benefits of bone protection therapies. In addition, it may be of value to explore experiences of delivering fracture prevention in other care settings. This would provide information on how to deliver services effectively elsewhere.

Acknowledgments The Refresh study group consists of Dr Andrew David Judge, Dr Muhammad Kassim Javaid, Professor Nigel Arden, Professor Cyrus Cooper, Professor Andrew Farmer, Dr Daniel PrietoAlhambra, Dr Jose Leal, Professor Michael Goldacre, Professor Alastair Gray, Dr Janet Lippett, Dr Rachael Gooberman-Hill and Laura Graham.

\section{Compliance with ethical standards}

HS\&DR Funding This project was funded by the National Institute for Health Research Health Services and Delivery Research Programme (project number 11/1023/01). We also wish to acknowledge the NIHR Musculoskeletal Biomedical Research Unit for the financial support.

Department of health disclaimer The views and opinions expressed therein are those of the authors and do not necessarily reflect those of the HS\&DR Programme, NIHR, NHS or the Department of Health.

Conflicts of interest AJ has received consultancy, lecture fees and honoraria from Servier, UK Renal Registry, Oxford Craniofacial Unit, IDIAP Jordi Gol, Freshfields Bruckhaus Deringer, has held advisory board positions (which involved receipt of fees) from Anthera Pharmaceuticals, INC., and received consortium research grants from ROCHE.

MKJ has received in the last 5 years honoraria for travel expenses, speaker fees and/or advisory committees from Lilly UK, Amgen, Servier, Merck, Medtronic, Internis and Consilient Health. He also serves on the Scientific Committee of the National Osteoporosis Society and International Osteoporosis Foundation.

$\mathrm{CC}$ has received consultancy, lecture fees and honoraria from AMGEN, GSK, Alliance for Better Bone Health, MSD, Eli Lilly, Pfizer, Novartis, Servier, Medtronic and Roche.

$\mathrm{SD}, \mathrm{AF}$ and RGH have no conflicts of interest to report. 
Ethical approval Ethical approval was provided by the Central University Research Ethics Committee (CUREC) in 2012, reference number MSD-IDREC-C1-2012-147. Each involved NHS Trust provided R\&D approval. Written, informed consent was obtained from all individual participants included in the study.

Open Access This article is distributed under the terms of the Creative Commons Attribution-NonCommercial 4.0 International License (http://creativecommons.org/licenses/by-nc/4.0/), which permits any noncommercial use, distribution, and reproduction in any medium, provided you give appropriate credit to the original author(s) and the source, provide a link to the Creative Commons license, and indicate if changes were made.

\section{References}

1. British Orthopaedic Association (2007) The care of patients with fragility fractures

2. Dennison E, Mohamed MA, Cooper C (2006) Epidemiology of osteoporosis. Rheum Dis Clin N Am 32(4):617-629

3. Chesser TJS, Handley R, Swift C (2011) New NICE guideline to improve outcomes for hip fracture patients. Injury 42(8):727-729

4. Johnell $\mathrm{O}$ et al (2004) Fracture risk following an osteoporotic fracture. Osteoporos Int 15(3):175-179

5. Abrahamsen B et al (2009) Excess mortality following hip fracture: a systematic epidemiological review. Osteoporos Int 20(10):1633-1650

6. Knopp J et al (2005) Calcitonin for treating acute pain of osteoporotic vertebral compression fractures: a systematic review of randomized, controlled trials. Osteoporos Int 16(10):1281-1290

7. Black DM et al (1999) Prevalent vertebral deformities predict hip fractures and new vertebral deformities but not wrist fractures. J Bone Miner Res 14(5):821-828

8. NICE (2008) Technology appraisal (TA) 161. Osteoporosis- - secondary prevention including strontium ranelate

9. Scottish Intercollegiate Guidelines Network (2009) Management of hip fracture in older patients. A National Clinical Guideline 111

10. NICE (2004) Clinical guideline 21. Clinical practice guideline for the assessment and prevention of falls in older people

11. Akesson $\mathrm{K}$ et al (2013) Capture the fracture: a best practice framework and global campaign to break the fragility fracture cycle. Osteoporos Int 24(8):2135-2152

12. Marsh D et al (2011) Coordinator-based systems for secondary prevention in fragility fracture patients. Osteoporos Int 22(7): 2051-2065

13. Drew $\mathrm{S}$ et al (2014) Describing variation in the delivery of secondary fracture prevention after hip fracture: an overview of 11 hospitals within one regional area in England. Osteoporos Int

14. Sumner M (2006) Qualitative research. The SAGE Dictionary of Social Research Methods. SAGE Publications, Ltd. London, England: SAGE Publications, Ltd. 249-251
15. Ayres L (2008) The SAGE encyclopedia of qualitative research methods. SAGE publications, Inc. SAGE Publications, Inc, Thousand Oaks, CA, pp 811-812

16. Braun V, Clarke V (2006) Using thematic analysis in psychology. Qual Res Psychol 3(2):77-101

17. Drew $S$ et al (2015) Implementation of secondary fracture prevention services after hip fracture: a qualitative study using extended normalization process theory. Implement Sci 10(1):57

18. ATKINSON R, Flint J (2003) Sampling, snowball: accessing hidden and hard-to-reach populations, The A-Z of Social Research. SAGE Publications, Ltd, London, England, pp 275-281

19. Ritchie J, Lewis J (eds) (2003) Qualitative research practice: a guide for social science students and researchers. Sage, London

20. NICE (2005) NICE Technology Appraisal 87: Bisphosphonates (alendronate, etidonate or risedronate), selective oestrogen receptor modulators (raloxifene) and parathyroid hormone (teriparatide) for the secondary prevention of osteoporotic fragility fractures in post menopausal women

21. NICE (2010) NICE technology appraisal guidelines 204: Denosumab for the prevention of osteoporotic fractures in postmenopausal women

22. Directorate, H.a.S.C. (2014) Indicators process guide. National Institute for Health and Care Excellence

23. Department of Health (2006) The musculoskeletal services framework

24. Papaioannou A et al (2007) Patient adherence to osteoporosis medications: problems, consequences and management strategies. Drugs Aging 24(1):37-55

25. Boonen S et al (2012) Time to onset of antifracture efficacy and year-by-year persistence of effect of zoledronic acid in women with osteoporosis. J Bone Miner Res 27(7):1487-1493

26. Lee S, Glendenning P, Inderjeeth CA (2011) Efficacy, side effects and route of administration are more important than frequency of dosing of anti-osteoporosis treatments in determining patient adherence: a critical review of published articles from 1970 to 2009. Osteoporos Int 22(3):741-753

27. Silverman SL et al (2015) Persistence at 12 months with denosumab in postmenopausal women with osteoporosis: interim results from a prospective observational study. Osteoporos Int 26(1):361-372

28. Clowes JA, Peel NFA, Eastell R (2004) The impact of monitoring on adherence and persistence with antiresorptive treatment for postmenopausal osteoporosis: a randomized controlled trial. J Clin Endocrinol Metab 89(3):1117-1123

29. Mitchell PJ (2013) Best practices in secondary fracture prevention: fracture liaison services. Curr Osteoporos Rep 11(1):52-60

30. Ganda K et al (2013) Models of care for the secondary prevention of osteoporotic fractures: a systematic review and meta-analysis. Osteoporos Int 24(2):393-406

31. Eisman JA et al (2012) Making the first fracture the last fracture: ASBMR task force report on secondary fracture prevention. J Bone Miner Res 27(10):2039-2046

32. Baker S.E, Edwards R (2012) How many qualitative interviews is enough? Nat Centre Rese Methods 